\title{
Estandarización y evaluación de ELISA para el serodiagnóstico de leishmaniasis I. Leishmaniasis cutánea
}

\author{
Augusto Corredor ${ }^{1}$, Myriam C. López², Sofía Duque ${ }^{3}$, Luis E. Gualdrón', Roberto B. Tesh ${ }^{5}$
}

\begin{abstract}
Resumen
Se evaluó la prueba inmunoenzimática ELISA para la detección de anticuerpos en pacientes con leishmaniasis cutánea, usando como conjugado anti-lgG humana con fosfatasa alcalina. La concentración óptima de antígeno, extracto de promastigotes de Leishmania, fue $5 \mu \mathrm{g} / \mathrm{mL}$ y las diluciones óptimas de los sueros tanto reactivos como no reactivos y de conjugado fueron $1: 400$ y 1:10.000, respectivamente. Se analizaron 163 sueros de pacientes que no habían estado en contacto con el parásito (Leishmania), 31 sueros de pacientes con leishmaniasis cutánea comprobada parasitológicamente y 39 sueros de pacientes con enfermedad de Chagas comprobada serológicamente.

Con valores de densidad óptica iguales o mayores de 0,502 la prueba mostró una especificidad del $94,6 \%$ y una sensibilidad del $58,1 \%$ debido a que los sueros de las personas con enfermedad de Chagas mostraron franca reacción cruzada con el antígeno de Leishmania. Sin embargo, el ELISA con un punto de corte de 0,314 , dado únicamente por los suerus de personas que no habían estado en contacto con el parásito, arroja una sensibilidad y especificidad del $83,9 \%$ y $83,2 \%$, respectivamente. Los resultados del estudio sugieren que el ELISA puede utilizarse en áreas donde la enfermedad de Chagas no sea endémica y las cuales están bien delimitadas en Colombia.
\end{abstract}

\section{Summary}

An ELISA test with alkaline phosphatase enzyme conjugate was evaluated to detect antibodies against Leishmania in patients with cutaneous leishmaniasis. The conjugate dilution was 1:10,000, the serum dilution 1:400 and the optimal antigen concentration 5 $\mu \mathrm{g} / \mathrm{mL}$. We analized 163 normal control sera, 31 sera from patients with a diagnosis of cutaneous leishmaniasis confirmed by parasitological methods and 39 sera from patients with Chagas' disease. The specificity of the test was $94.6 \%$ and its sensitivity $58.1 \%$ with optical density values equal or greater than 0.502 . Cross-reactivity was detected with sera from Chagas' disease patients. However, the specificity and sensitivity of the test were $83.2 \%$ and $83.9 \%$, respectively with OD values equal or greater than 0.314 obtained from normal control sera values. The results of this study suggest that ELISA might be a useful test in diagnosing cutaneous leishmaniasis in areas where Chagas' disease is not endemic, which are very well located in Colombia.

Médico Cirujano; Director, Instituto de Salud en el Trópico, Facultad de Medicina, Universidad Nacional de Colombia, Santafé de Bogotá, D.C.

2 Bacterióloga, Laboratorio de Parasitología, Instituto Nacional de Salud, Santafé de Bogotá, D.C.

3 Bióloga, M.Sc. en Parasitología Médica, Laboratorio de Parasitología, Instituto Nacional de Salud, Santafé de Bogotá, D.C.

4 Biólogo, Laboratorio de Parasitología, Instituto Nacional de Salud, Santafé de Bogotá, D.C.

5 Médico, Departamento de Epidemiología y Salud Pública, Escuela de Medicina, Universidad de Yale, Estados Unidos de América. 
La leishmaniasis cutánea americana es una enfermedad transmitida por flebotomíneos (Lutzomyia) y producida por las especies de los subgéneros Leishmania y Viannia, las cuales se encuentran distribuidas tanto en Centroamérica como en Suramérica (1). En Colombia es una de las enfermedades tropicales que reviste un problema de salud pública. El serodiagnóstico de la leishmaniasis cutánea se ha llevado a cabo mediante: fijación de complemento (2), aglutinación directa $(3,4)$, hemaglutinación pasiva $(5)$, inmunofluorescencia (6-12) y ELISA, la cual desde su publicación (13) ha sido adaptada como serodiagnóstico de una gran variedad de enfermedades (14-16).

El presente trabajo tuvo por objeto estandarizar y evaluar el ELISA para ser utilizado en el serodiagnóstico de leishmaniasis cutánea.

\section{Materiales y métodos}

Parásitos: promastigotes de las cepas $\mathrm{MHOM} /$ CO/84/CL020 y MHOM/CO/82/CL01, aislados de pacientes con leishmaniasis cutánea e identificadas como Leishmania panamensis y Leishmania amazonensis, respectivamente (17), fueron empleados para la preparación del antígeno soluble. Los cultivos en masa de estas cepas fueron llevados a cabo en REI modificado (18).

Preparación del antígeno: el antígeno soluble se preparó de acuerdo a la metodología descrita por Pappas y col. (19), modificando a nueve veces el proceso de congelación a $-196^{\circ} \mathrm{C}$ y descongelación a $4^{\circ} \mathrm{C}$ de los parásitos. Estos se sonicaron tres veces después de la tercera, sexta y novena congelada y descongelada, en condiciones de refrigeración a $20 \mathrm{kHz}$ durante quince segundos. Se determinó la concentración de proteínas por el método de Lowry (20) y el antígeno se almacenó en alícuotas de $0,5 \mathrm{~mL}$ a $-70^{\circ} \mathrm{C}$.

Muestras: se analizaron 233 muestras de suero: 163 negativas para leishmaniasis y enfermedad de Chagas procedentes de Barú (Bolívar), región donde estas enfermedades no se presentan debido a la ausencia de sus vectores, Lutzomyia y triatomíneos domiciliarios (A. Morales y M.C. Ferro, comunicación personal), 31 positivas de pacientes con leishmaniasis comprobada parasitológicamente y 39 de personas de zonas donde es prevalente la enfermedad de Chagas y no se encuentra leishmaniasis y que tenían anticuerpos contra Trypanosoma cruzi a títulos mayores o iguales de 1:32 por inmunofluorescencia indirecta. Estos últimos sueros se tomaron como posible reacción cruzada.

Conjugado enzimático: se usó el conjugado anti-IgG humano con fosfatasa alcalina preparado en el Laboratorio de Inmunología del instituto Nacional de Salud.

Inmunoensayo enzimático: el ELISA se llevó a cabo de acuerdo con las normas establecidas por Voller y col. (21), empleando microplacas de poliestireno Dynatech M-129-A y un fotocolorímetro Uniskan I para la lectura.

La concentración óptima de antígeno se determinó por titulación de éste en un rango de 0,76 a $10 \mu \mathrm{g} / \mathrm{mL}$. El antígeno se disolvió en una solución carbonato-bicarbonato de sodio $0,1 \mathrm{M}$, $\mathrm{pH} 9,6$. Se incubó tres horas a temperatura ambiente en las microplacas agregando $100 \mu \mathrm{L}$ por pozo. Después de lavar tres veces con solución salina reguladora de fosfatos (PBS) $0,15 \mathrm{M}, \mathrm{pH}$ 7,4 con Tween 20 al 0,05\%, se agregaron $100 \mu \mathrm{L}$ de suero en un rango de diluciones de 1:25 a 1:800 y se incubó tres horas a temperatura ambiente. Se lavó nuevamente y se agregaron $100 \mu \mathrm{L}$ de conjugado enzimático, el cual se había titulado previamente con un suero reactivo y uno no reactivo a la dilución 1:10.000; éste se incubó 18 horas a $4^{\circ} \mathrm{C}$. Se repitió el proceso de lavado como se describió anteriormente y luego se agregaron $100 \mu \mathrm{L}$ de paranitro-fenil-fosfato $1 \mathrm{mg} /$ $\mathrm{mL}$ en solución reguladora de dietanolamina al $10 \%, \mathrm{pH} 9,8$. Se incubó treinta minutos $y$, finalmente, se agregaron $25 \mu \mathrm{L}$ de $\mathrm{NaOH} 3 \mathrm{~N}$. Se leyó la densidad óptica a una longitud de onda de $405 \mathrm{~nm}$.

\section{Análisis estadístico}

1. Determinación del punto de corte: el valor de densidad óptica necesario para diferenciar las muestras positivas de las negativas, se estableció calculando el promedio más dos desviaciones estándar $(p<0,05)$ de los valores 
de densidad óptica de las muestras de personas sanas (22).

2. Determinación de los parámetros de la prueba: la sensibilidad (S), especificidad (E), valor predictivo positivo (VPP) y valor predictivo negativo (VPN) del ELISA se determinaron utilizando una tabla de contigencia de $2 \times 2$ (23). Los intervalos de confianza exactos para la sensibilidad y especificidad, se determinaron mediante las tablas científicas del documento Geigy (24).

\section{Resultados}

\section{Estandarización del ELISA}

Concentración de antígeno: se determinó con sueros positivos y negativos para leishmaniasis frente a concentraciones variables de antígeno, tomándose como concentración óptima la densidad óptica a la cual se encontró una diferencia significativa entre la muestra positiva y la negativa. La concentración óptima de antígeno se estableció en $5 \mu \mathrm{g} / \mathrm{mL}$ (figura 1).

Valor de dilución del suero: la dilución óptima de los sueros fue 1:400 que es aquélla donde se observó una buena diferenciación entre positivo y negativo (figura 2).

\section{Evaluación del ELISA}

\section{Punto de corte}

Serodiagnóstico en cualquier área geográfica: el punto de corte para el ELISA del estudio fue de 0,502 . Las muestras se consideraron positivas si la densidad óptica era igual o mayor a la media más dos desviaciones estándar obtenida de las muestras negativas para leishmaniasis y de las muestras de personas de zonas donde es prevalente la enfermedad de Chagas, consideradas como de reacción cruzada.

Sueros con valores de densidad óptica mayores o iguales a 0,502 se consideraron positivos y sueros con densidades ópticas menores a 0,501 se consideraron negativos.

Serodiagnóstico en áreas geográficas donde la enfermedad de Chagas no sea endémica: se estableció un segundo punto de corte del ELISA que sería el opcional para el serodiagnóstico de

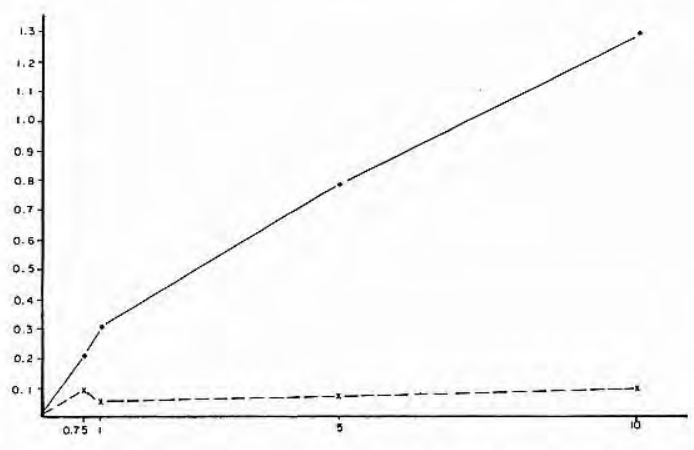

Figura 1. Concentración óptima de antígeno de Leishmania. Eje X: concentración de antígeno $\mu \mathrm{g} / \mathrm{mL}$ Eje Y: densidad óptica (405 nm) o: muestra positiva $\mathrm{x}$ : muestra negativa

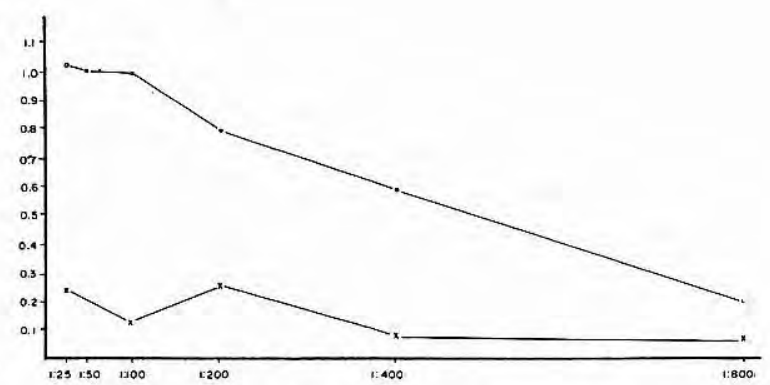

Figura 2. Dilución óptima deL suero.

Eje $X$ : diluciones de suero

Eje Y: densidad óptica (405 nm)

o: muestra positiva

$\mathrm{x}$ : muestra negativa

leishmaniasis cutánea donde la enfermedad de Chagas no sea endémica. El punto de corte se calculó como se mencionó anteriormente, pero teniendo en cuenta solamente las muestras negativas para leishmaniasis y enfermedad de Chagas. Así, los sueros con valores de densidad óptica mayores o iguales a 0,314 se consideraron positivos y sueros con valores de densidad óptica menores a 0,313 se consideraron negativos.

\section{Especificidad y sensibilidad}

Serodiagnóstico en cualquier área geográfica: con un punto de corte de 0,502 se determinó que de 31 pacientes con leishmaniasis confirmada parasitológicamente 18 fueron positivos y 13 fueron falsos negativos con la prueba, obteniéndose una sensibilidad del $58,1 \%$ (tabla 1 ). 
De 202 pacientes negativos para las enfermedades de leishmaniasis y Chagas y de personas de zonas donde es prevalente la enfermedad de Chagas, 191 fueron negativos y 11 fueron falsos positivos, resultando una especificidad del $94,6 \%$ (tabla 1).

\section{Reacción cruzada}

Treinta y nueve sueros de pacientes con enfermedad de Chagas, confirmada serológicamente, se emplearon como grupo control y se procesaron con antígeno de Leishmania. En este caso se observó una sensibilidad del $58,1 \%$ y una especificidad del $71,8 \%$, menor que la obtenida con sueros negativos para leishmaniasis y enfermedad de Chagas.

El $94,6 \%$ (191/202) de los sueros negativos, el $41,9 \%$ de los sueros positivos para leishmaniasis y el $71,8 \%$ (28/39) de los sueros con anticuerpos contra Trypanosoma cruzi presentaron valores menores a 0,501 (tablas 1y 2).

Serodiagnóstico en áreas geográficas donde la enfermedad de Chagas no se endémica: con un punto de corte de 0,314 se demostró que de los 31 pacientes con leishmaniasis confirmada parasitológicamente, 26 fueron positivos y 5 fueron falsos negativos, obteniéndose así una sensibilidad del $83,9 \%$ (tabla 3 ).

De 202 pacientes negativos para las enfermedades de leishmaniasis y Chagas y de personas de

Tabla 1. Vaior diagnóstico de ELISA en leishmaniasis cutánea. Grupo control Barú, pacientes negativos para las enfermedades de leishmaniasis y Chagas y pacientes con enfermedad de Chagas confirmada serológicamente.

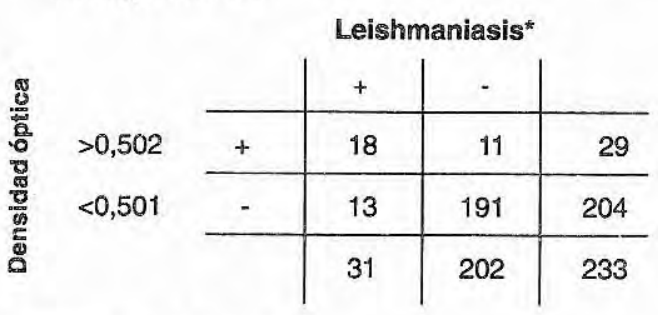

S: $58,1 \%$; rango: $(39,1-75,5)$

E: $94,6 \%$; rango: $(91,0-97,6)$

VPP: $62,1 \%$

VPN: $93,6 \%$

* parasitológicamente confirmada zonas donde es prevalente la enfermedad de Chagas, 168 fueron negativos y 34 fueron falsos positivos, para una especificidad del $83,2 \%$ (tabla 3).

Tabla 2. Valor diagnóstico de ELISA en leishmaniasis cutánea. Grupo control: pacientes serológicamente positivos para Chagas.

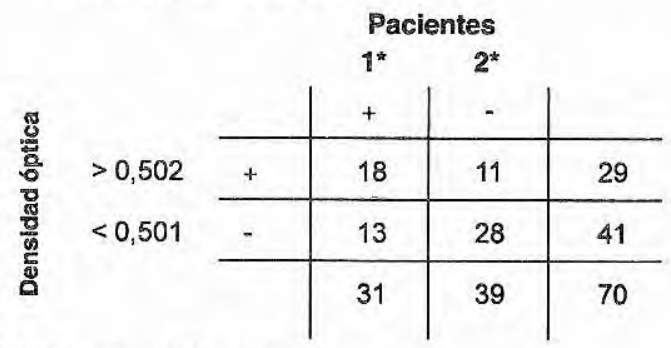

S: $58,06 \%$; rango $(39,1-75,5)$

E: $71,8 \%$; rango $(55,1-85,0)$

VPP: $62,1 \%$

VPN: $68,3 \%$

$1^{*}$ con leishmaniasis cutánea confirmada parasitológicamente $2^{*}$ con enfermedad de Chagas

Tabla 3. Valor diagnóstico de ELISA en leishmaniasis cutánea. Grupo control Barú, pacientes negativos para las enfermedades de leishmaniasis y Chagas.

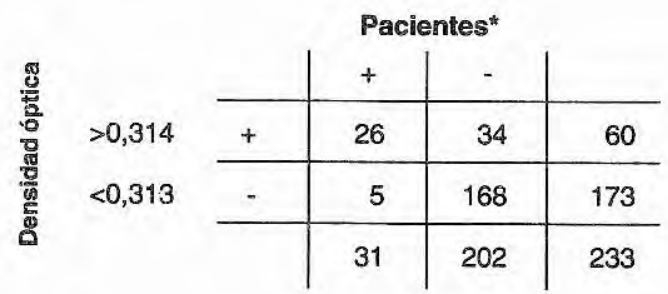

S: $83,9 \%$; rango $(66,3-94,6)$

E: $83,2 \%$; rango $(79,3-89,6)$

VPP: $43,3 \%$

VPN: $97,1 \%$

* leishmaniasis parasitológicamente confirmada

\section{Discusión}

Las pruebas empleadas para el serodiagnóstico de una enfermedad dependen tanto de factores intrínsecos a la prueba como de aquéllos de la virulencia de la especie infectante y de los inmunológicos inherentes al huésped que sufre la enfermedad. Los factores intrínsecos a las pruebas pueden optimizarse con el fin de ofrecer las mejores condiciones para la estandarización y evaluación de la prueba; pero, los factores 
dependientes de la especie infectante o los inherentes al huésped influyen en el éxito o el fracaso de la aplicación de una prueba para la confirmación de la enfermedad.

La prueba ELISA estandarizada y evaluada para el serodiagnóstico de leishmaniasis cutánea en el presente estudio para cualquier área geográfica, presentó una sensibilidad del $58,1 \%$ y una especificidad del $94,6 \%$.

El $58,1 \%$ de sensibilidad de la prueba puede deberse a factores dependientes de la respuesta inmune del huésped representada en un bajo tenor de anticuerpos en los sueros que los pacientes con leishmaniasis cutánea suelen desarrollar frente a la infección con Leishmania (25) y los cuales pueden atribuirse a la especie de Leishmania infectante, la duración de la infección, el tipo y tamaño de las lesiones y, también, a factores genéticos del huésped como mayor o menor susceptibilidad a la infección (12).

En el presente estudio, la sensibilidad del ELISA se redujo de un $83,9 \%$ con punto de corte del 0,314 , al $58,1 \%$ con punto de corte del 0,502 , debido a que éste se estableció utilizando los valores de los sueros negativos para leishmaniasis y enfermedad de Chagas lo que indica que para el serodiagnóstico de leishmaniasis mediante ELISA, en zonas chagásicas, se requiere de una mayor cantidad de anticuerpos circulantes en los pacientes con leishmaniasis cutánea lo cual depende de la respuesta inmune del huésped.

Los anticuerpos desarrollados contra Trypanosoma, en los pacientes con enfermedad de Chagas, reaccionan inespecíficamente frente a los antígenos de Leishmania. Esto se debe a que los parásitos del género Leishmania poseen antígenos comunes con otros flagelados de la familia Trypanosomatidae como el Trypanosoma y los cuales interfieren con el serodiagnóstico de la leishmaniasis cutánea (16). Tales reacciones interfieren con el establecimiento de los puntos de corte ya que para ello debe trabajarse con valores de densidad óptica altos $(0,502)$ afectando directamente el valor de sensibilidad de la prueba.
Las pruebas serológicas hasta ahora establecidas para la confirmación de la enfermedad han sido exitosas en el caso de la leishmaniasis visceral, ya que en ésta se presenta una franca respuesta inmunológica humoral del huésped y la presencia de anticuerpos circulantes es lo suficientemente óptima para ser detectados por cualquiera de las pruebas hasta ahora desarrolladas; mientras que ELISA, una de las mejores pruebas para la mayoría de los inmunodiagnósticos de enfermedades que ofrece alta sensibilidad y especificidad, sería menos útil para la confirmación de la leishmaniasis cutánea como han observado Voller y col. (21) y como se informa en el presente trabajo.

Sin embargo, se propone que el ELISA del presente trabajo con un punto de corte de 0,314 que demuestra una sensibilidad y una especificidad del $83,9 \%$ y $83,2 \%$, respectivamente, puede utilizarse como herramienta inmunodiagnóstica en áreas donde la enfermedad de Chagas no sea endémica, las cuales están muy bien delimitadas en Colombia.

Se conoce que la distribución de la enfermedad de Chagas y de los triatomíneos domiciliarios Rhodnius prolixus, Triatoma dimidiata, Triatoma maculata, Rhodnius robustus, Rhodnius brethesi y Triatoma venosa, está exclusivamente en la región oriental del país por debajo de los 2.000 msnm y que está constituida por la vertiente oriental del macizo central del río Magdalena, la cordillera oriental y los llanos orientales (26).

Así, el ELISA estandarizado y evaluado en el presente trabajo se puede utilizar en el occidente de Colombia donde la enfermedad de Chagas está ausente y existe una gran densidad de población expuesta a la infección con especies de L. mexicana, L. braziliensis y, principalmente, L. panamensis. Sin embargo, debe enfatizarse que en todos los casos sospechosos de leishmaniasis debe intentarse la confirmación parasitológica.

\section{Agradecimientos}

Los autores desean expresar sus agradecimientos a las siguientes personas del Instituto Nacional de Salud, que de una u otra forma colaboraron para que se realizara este estudio: 
doctor Luis Carlos Orozco, Laboratorio de Patología; doctor Rubén Santiago Nicholls, Coordinador de Investigación, Laboratorio de Parasitología; Carmenza Murillo y Dioselina Peláez. Al doctor Ricardo Muñoz y colaboradores por las muestras de sueros de los pacientes de Barú.

Este estudio se realizó con los auspicios del Instituto Nacional de Salud de Colombia y del Grant Al-2018 del National Institute of Health, U.S.A.

\section{Referencias}

1. Dedet JP. Leishmania et leishmanioses du continent américain. Ann Inst Pasteur 1993;4:3-25.

2. Moses A. De fixação de complemento na leishmaniasis tegumentares. Bull inst Pasteur 1919;17:530.

3. Senekjie HA, Lewis RA. Diagnosis of leishmaniasis by slide agglutination. Proc Soc Exp Biol Med 1944;57:17-9.

4. Allain DS, Kagan IG. A direct agglutination test for leishmaniasis. Am J Trop Med Hyg 1975;24:232-6.

5. Bray RS, Lainson R. Studies on the immunology and serology of leishmaniasis. V. The use of particles as vehicles in passive agglutination tests. Trans R Soc Trop Med Hyg 1967;61:490-505.

6. Oddo FG, Cascio G. Il test di immunofluorescenza nelle leishmaniosi viscerale e cutanea. Riv Ist Sieroter Ital 1963;38:139-45.

7. Bray RS, Lainson R. The immunology and serology of leishmaniasis. I. The fluorescent antibody staining technique. Trans R Soc Trop Med Hyg 1965;59:534-44.

8. Quilici M, Dunan S, Ranque J. L'immunofluorescense dans les leishmanioses. Comparaison avec la reaction de fixation du complément. Med Trop 1968;28:37-43.

9. Bittencourt AL, Sodré A, Andrade ZA. Pesquisa de anticorpos circulantes pelo método de imunofluoreacencia na leishmaniose tegumentar. Rev Inst MedTrop Sao Paulo 1968;10:247-52.

10. Walton $\mathrm{BC}_{5}$ Brooks WH, Arjona I. Serodiagnosis of American leishmaniasis by indirect fluorescent antibody test. Am J Trop Med Hyg 1972;21:296-99.

11. Matossian RM, Kurban AK, Malak JA. Circulating antibodies in cutaneous leishmaniasis: their detection by immunofluorescense. Trans R Soc Trop Med Hyg 1975;69:450-2.

12. Pappas MG, McGreevy PB, Hajkowski $R$, Hendricks LD, Oster ChN, Hockmeyer WT. Evaluation of promastigote and amastigote antigens in the indirect fluorescent antibody test for american cutaneous leishmaniasis. Am J Trop Med Hyg 1983;32:1260-7.

13. Engvall E, Jonnson K, Perlmann P. Enzyme-linked immunosorbent assay. II. Quantitative assay of protein antigen immunoglobulin $G$ by means of enzyme-labelled antigen and antigen-coated tubes. Biochem Biophys Acta 1971;251:427-34.

14. Hommel M, Peters W, Ranque J, Quilici M, Lanotte G. The micro-ELISA technique in the serodiagnosis of visceral leishmaniasis. Ann Trop Med Parasitol 1978;72:2138.

15. Edrissian GH, Darabian P. A comparison of enzymelinked immunosorbent assay and indirect fluorescent antibody test in the serodiagnosis of cutaneous and visceral leishmaniasis in Iran. Trans R Soc Trop Med Hyg 1979;73:289-93.

16. Roffi J, Dedet JP, Desjeux P, Garré MT. Detection of circulating antibodies in cutaneous leishmaniasis by enzymelinked immunosorbent assay (ELISA). Am J Trop Med Hyg 1980;29:183-9.

17. Corredor A, Kreutzer RD, Tesh RB, et al. Distribution and etiology of leishmaniasis in Colombia.Am J Trop Med Hyg 1990;42:206-14.

18. Duque S, Cáceres E, Corredor A. Comportamiento de flagelados de la familia Trypanosomatidae en dos medios de cultivo modificados. Biomédica 1988;8:21-7.

19. Pappas MG, Hajkowski R, Cannon LT Sr, Hockmeyer WT. Dot enzyme-linked immunosorbent assay (DOTELISA): comparison with standard ELISA and complement fixation assays for the diagnosis of human visceral leishmaniasis. Vet Parasitol 1984;14:239-49.

20. Lowry OH, Rosebrough NJ, Farr AL, Randall RJ. Protein measurement with the folin phenol reagent. $J$ Biol Chem 1951;193:265-75.

21. Voller A, Bartlett A, Bidwell DE. Enzyme immunoassays for parasitic diseases. Trans R Soc Trop Med Hyg 1976;70:98-106.

22. Kurstak E. Progress in enzyme immunoassays: production of reagents, experimental desing, and interpretation. Bull WHO 1985;63:793-809.

23. Griner PF, Mayewski RJ, Mushlin Al, Greenland P. Selection and interpretation of diagnostic tests and procedures. Ann Intern Med 1981;94:553-600.

24. Geigy JR. Documento Geigy. Tablas científicas. Sexta edición. Basilea: Suiza.

25. Araujo FG, Mayrink W. Fluorescent antibody test in visceral leishmaniasis. II. Studies on the specificity of the test. Rev Inst Med Trop Sao Paulo 1968;10:41-5.

26. Corredor A, Santacruz MM, Páez S, Guatame LA. Distribución de los triatomíneos domiciliarios en Colombia. Bogotá,D.C.: Instituto Nacional de Salud, 1990:9. 